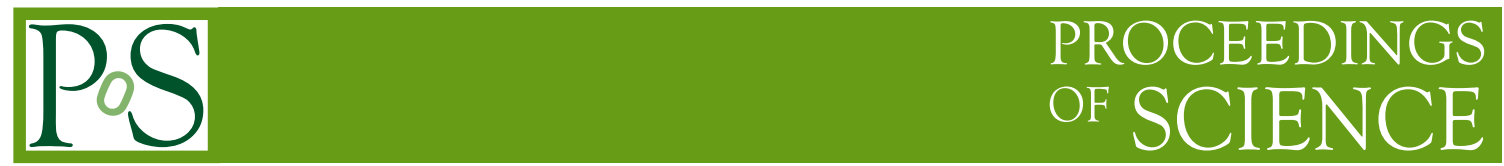

\title{
FLAIR, a next-generation facility for low-energy antiprotons
}

\author{
Eberhard Widmann*广 \\ Stefan Meyer Institute, Vienna, Austria \\ E-mail: eberhard.widmanneoeaw.ac.at
}

The Facility for Low-energy Antiproton and Ion Research FLAIR is an addition to the FAIR facility at Darmstadt, promising antiproton beams of lower energies and higher intensities than currently available. Both fast and slow extracted beams are planned. FLAIR requires in its original design the NESR to decelerate antiprotons stacked in the RESR and is thus not part of the modularized start version of FAIR.

8th International Conference on Nuclear Physics at Storage Rings-Stori11,

October 9-14, 2011

Laboratori Nazionali di Frascati dell'INFN, Italy

\footnotetext{
*Speaker.

${ }^{\dagger}$ for the FLAIR collaboration http: //www.gsi.de/flair
} 


\section{Overview}

The FLAIR facility as layed out in its Conceptual Design Report [1] includes as a main component a production facility for antiprotons aimed at generating high-energy $(1-15 \mathrm{GeV})$ antiprotons in the HESR storage ring for the PANDA experiment (cf. Fig. 1). It was realized early that the also contained NESR storage ring originally planned to be used for in-ring atomic and nuclear physics experiments could be also used to decelerate antiprotons to $\sim 30 \mathrm{MeV}$ kinetic energy. A letter of intent [2] was submitted for a low-energy facility called FLAIR. After acceptance, FLAIR became part of the core FAIR facility which was documented by including the FLAIR Technical Proposal [3] into the FAIR Baseline Technical Report [4].

When the FAIR Modularized Start Version was decided in 2010, the NESR and consequently FLAIR were not part because of lack of funding and thus both can be built only if - after completion and initial operation of the start version - additional funding can be raised. Within this scenario FLAIR could start earliest in the first half of the next decade.

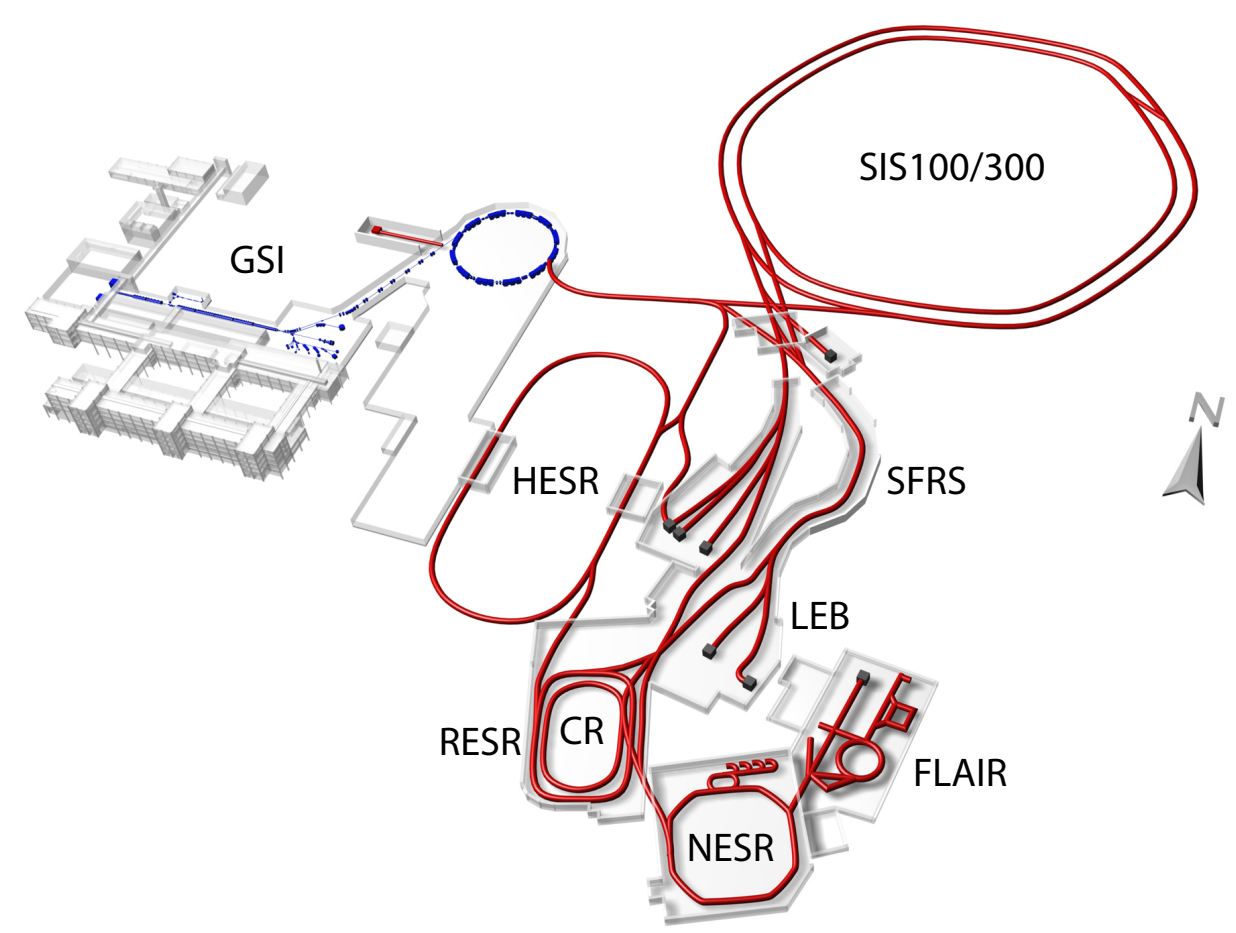

Figure 1: Overview of the FAIR accelerators. Existing GSI accelerators are marked in blue, new FAIR ones in red.

\section{The FLAIR facility}

FLAIR was designed to be a next-generation low-energy antiproton facility providing both fast and slow extracted cooled beams at two orders of magnitude lower energy and two orders of magnitude higher intensity than the currently existing Antiproton Decelerator (AD) of CERN. A 


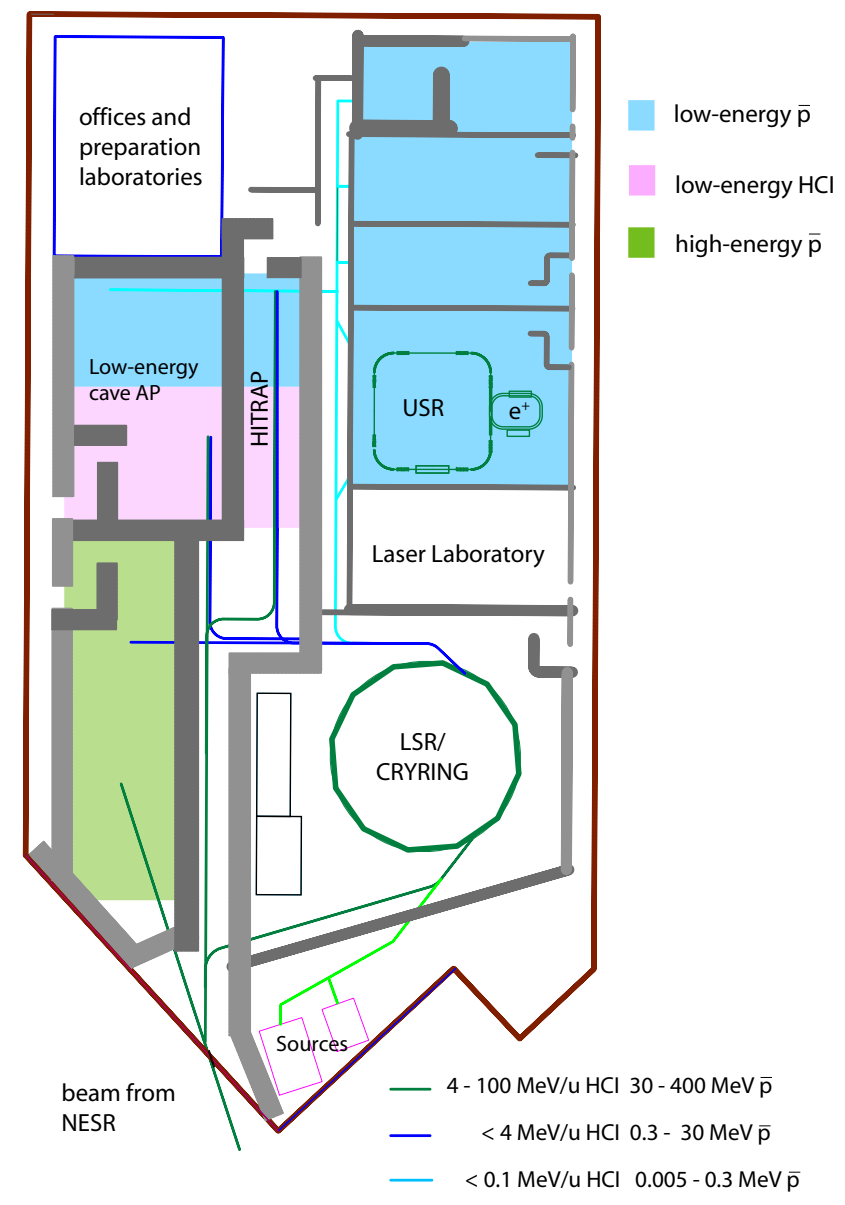

Figure 2: Layout of the FLAIR facility

key feature is that all installations can be equally well used for antiprotons and highly charged ions (HCI).

The FLAIR facility contains three major components

LSR The Low Energy Storage Ring LSR takes the beam from NESR at $30 \mathrm{MeV}$ kinetic energy for antiprotons. Using electron cooling it can provide both fast and slow extracted beams of antiprotons between $30 \mathrm{MeV}$ and $300 \mathrm{keV}$ energy.

USR The Ultra low-energy Storage Ring USR is an electrostatic ring capable of decelerating $\overline{\mathrm{p}}$ down to $20 \mathrm{keV}$. Like LSR it contains an electron cooler and can provide fast and slow extracted beams.

HITRAP The HITRAP facility [5, 6] is designed to trap and cool highly charged ions in a Penning trap, from where they can be extracted to several experiments. It is currently being built up at the ESR facility of GSI. At FLAIR it will receive HCI directly from the NESR and $\bar{p}$ from LSR. 
In addition an area for high-energy antiprotons up to $400 \mathrm{MeV}$ directly from NESR (green shaded area in Fig. 2) and an area for HCI also supplied from NESR ("Low-energy cave AP" in Fig. 2) are included, providing a variety of beams of both highly charged ions and antiprotons that will be unique.

For LSR, the CRYRING operating previously at Manne Siegbahn Laboratory (MSL) in Stockholm was chosen since it was going to be discontinued. MSL has already made the necessary modifications to allow for injection from NESR and extraction [7], and the ring will soon be available for use at FAIR. The USR is of similar design as the Cryogenic Storage Ring CSR under construction at MPI Heidelberg [8] and is being designed at the Cockcroft Institute in UK. With HITRAP going into operation at GSI, already two of the major components of FLAIR are available now, long before a realization of FLAIR will be possible in the layout described above.

\section{Physics program}

The physics program of FLAIR has been described in the letter of intent [2, 9] in 2004 with a start of the facility around 2010-2012 in mind. It consists of several topics:

1. precision spectroscopy of antiprotonic atoms and antihydrogen for tests of CPT symmetry,

2. atomic collisions: sub-femtosecond correlated dynamics: ionization, energy loss, antimattermatter collisions,

3. antiprotons as hadronic probes, and

4. medical applications.

The first topic is the main research theme at the AD of CERN. After the recent approval of the ELENA storage ring [10] to reduce the available energy to $100 \mathrm{keV}$ in order to increase the number of trapped antiprotons, this program will be continued at AD-ELENA for the next 10-15 years. Also a reaction microscope and a small electrostatic storage ring are being discussed for ELENA so that a part of the physics of item 2 will be performed at CERN.

Item 3, the use of $\bar{p}$ as hadronic probes, requires slow extracted beam and can therefore not be performed at AD-ELENA. Interesting topics are antiprotonic atoms which allow the study of neutron skins and halos of nuclei. This is one of the few methods that can directly determine neutron distributions inside nuclei and is thus of great interest to the nuclear physics community. The availability of unstable nuclei at the Low-energy Branch LEB of the Super FRagment Separator SFRS (see Fig. 1) just next door to FLAIR opens the unique possibility to extend these studies to unstable nuclei. A proposal for such an experiment called EXO+pbar has been made [11] and will require only an additional beam line between the LEB and FLAIR buildings.

The possible use of $\bar{p}$ for tumor therapy (item 4 ) is being tested by the ACE experiment at CERN. If a significant advantage of $\bar{p}$ over other particles (by the additional energy deposit through antiproton annihilation at the end of the $\overline{\mathrm{p}}$ range) will be confirmed, higher energy antiproton beams will be needed that are not available at AD-ELENA to perform further tests. FLAIR contains a high-energy cave which will be perfectly suited for such studies. 


\section{Outlook}

After the delay of FLAIR and the approval of ELENA at CERN, the low-energy antiproton physics community interested in precision tests of fundamental symmetries and interactions has secured a medium term future at CERN-AD. ELENA will increase the number of trapped antiprotons by $1-2$ orders of magnitude compared to the $\mathrm{AD}$, but will still produce only $1 / 10$ of the $\overline{\mathrm{p}}$ flux possible at FLAIR due to the lack of a dedicated ring for $\bar{p}$ stacking. This fact, the availability of slow extracted beams and a variety of energies for cooled beams of antiprotons and highly charged ions makes FLAIR still a very attractive facility.

Recently discussions on alternative scenarios to decelerate highly charged ions and antiprotons in other rings (RESR or CR) are being discussed, which would have the advantage to make these beams available much earlier, but not at the performance of the full FLAIR facility. The feasibility as well as the impact of these options needs to be carefully assessed before a scenario can be fixed.

\section{Acknowledgements}

The author would like to thank his colleagues from the FLAIR steering committee for several years of fruitful working together, especially H. Danared, Th. Stöhlker, W. Quint and D. Grzonka, and K. Blaum for taking over the spokespersonship for FLAIR from 2012.

\section{References}

[1] An international accelerator facility for beams of ions and antiprotons, conceptual Design Report, GSI, Nov. 2001.

[2] FLAIR - a facility for low-energy antiproton and ion research, Letter of Intent, Feb. 2004. available from http://www.gsi.de/flair/.

[3] FLAIR - a facility for low-energy antiproton and ion research, Technical Proposal, Jan. 2005. available from http://www.gsi.de/flair/.

[4] FAIR - Baseline Technical Report, Tech. Rep. ISBN: 3-9811298-0-6, FAIR, 2009.

[5] H.-J. Kluge et. al., HITRAP: A Facility at GSI for Highly Charged Ions, in Advances in Quantum Chemistry (S. Salomonson and E. Lindroth, eds.), vol. 53, pp. 83-98, Academic Press, 2008.

[6] F. Herfurt. these proceedings.

[7] H. Danared et. al., LSR Technical Design Report, Tech. Rep. available from http://www.gsi.de/flair/, Manne Siegbahn Laboratory, Stockholm, 2011.

[8] R. von Hahn, F. Berg, K. Blaum, J. C. Lopez-Urrutia, F. Fellenberger, M. Froese, M. Grieser, C. Krantz, K.-U. Kühnel, M. Lange, S. Menk, F. Laux, D. Orlov, R. Repnow, C. Schröter, A. Shornikov, T. Sieber, J. Ullrich, A. Wolf, M. Rappaport, and D. Zajfman, The electrostatic cryogenic storage ring csr-mechanical concept and realization, Nuclear Instruments and Methods in Physics Research Section B: Beam Interactions with Materials and Atoms 269 (2011), no. 242871 -2874 .

[9] E. Widmann, Plans for a next-generation low-energy antiproton facility, Physica Scripta 72 (Dec, 2005) C51-C56. 
[10] ELENA- an updated cost and feasibility study, Tech. Rep. CERN-BE-2010-029 OP, CERN, 2010.

[11] M. Wada and Y. Yamazaki Nucl. Instr. Meth. B 214 (2004) 196-200. 